\title{
In-Network Redundancy Generation for Opportunistic Speedup of Data Backup
}

\author{
Lluis Pamies-Juarez, Anwitaman Datta and Frédérique Oggier \\ Nanyang Technological University; Singapore
}

July 5, 2018

\begin{abstract}
Erasure coding is a storage-efficient alternative to replication for achieving reliable data backup in distributed storage systems. During the storage process, traditional erasure codes require a unique source node to create and upload all the redundant data to the different storage nodes. However, such a source node may have limited communication and computation capabilities, which constrain the storage process throughput. Moreover, the source node and the different storage nodes might not be able to send and receive data simultaneously - e.g., nodes might be busy in a datacenter setting, or simply be offline in a peer-to-peer setting - which can further threaten the efficacy of the overall storage process. In this paper we propose an "in-network" redundancy generation process which distributes the data insertion load among the source and storage nodes by allowing the storage nodes to generate new redundant data by exchanging partial information among themselves, improving the throughput of the storage process. The process is carried out asynchronously, utilizing spare bandwidth and computing resources from the storage nodes. The proposed approach leverages on the local repairability property of newly proposed erasure codes tailor made for the needs of distributed storage systems. We analytically show that the performance of this technique relies on an efficient usage of the spare node resources, and we derive a set of scheduling algorithms to maximize the same. We experimentally show, using availability traces from real peer-to-peer applications as well as Google data center availability and workload traces, that our algorithms can, depending on the environment characteristics, increase the throughput of the storage process significantly (up to $90 \%$ in data centers, and $60 \%$ in peer-to-peer settings) with respect to the classical naive data insertion approach.
\end{abstract}

Keywords: distributed storage systems, data insertion, locally repairable codes, self-repairing codes

\section{Introduction}

There is a continuous and rapid global growth in data storage needs. Archival and backup storage form a specific niche of importance to both businesses and individuals. A recent market analysis from IDd1 stated that the global revenue of the data archival business is expected to reach $\$ 6.5$ billion in 2015 . The necessity

\footnotetext{
* The research of L. Pamies-Juarez and F. Oggier is supported by the Singapore National Research Foundation under Research Grant NRF-CRP2-2007-03. A. Datta's work has been supported by A*Star TSRP grant number 102-158-0038.

${ }_{1}^{1}$ http://www . idc . com/getdoc . jsp? containerId=230762
} 
to cost-effectively scale-up data backup systems to meet this ever growing storage demand poses a challenge to storage systems designers.

When a large volume of data is involved, deploying a networked distributed storage system becomes essential, since a single storage node cannot scale. Furthermore, distribution provides opportunities for fault tolerance and parallelized I/O. Examples of such distributed storage systems are readily found in datacenter environments, including distributed file systems such as GFS [8] or HDFS [2], distributed key-value stores like Dynamo [10] or Cassandra [14, for storing huge volume of scientific or multimedia content 9, 17] as well as in ad-hoc end user resource based peer-to-peer (P2P) settings such as OceanStore [13] and friend-to-friend (F2F) storage systems [24, 15, a special kind of peer-to-peer systems often considered particularly suitable for personal data backup.

An important design aspect in distributed storage systems is redundancy management. Data replication provides a simple way to achieve high fault-tolerance, while erasure codes such as Reed-Solomon codes [22] are more sophisticated alternatives, capable of significantly reducing the data storage footprint for different levels of fault-tolerance [23, 16, 27, 25, 12. Various trade-offs in adopting erasure codes in storage systems, such as storage overhead \& fault-tolerance, access frequency \& decoding overheads, but also due to the need of replenishment of lost redundancy, repair bandwidth \& repair time after failures, have been studied in the literature, revealing in particular that erasure codes are particularly suited for backup and archival storage, where data access is infrequent, and hence the effects of decoding are marginal. Consequently, many modern data center oriented storage and file systems such as Microsoft's Azure [11, HDFS [28] and the next generation of Google's file system (Colossus) have incorporated erasure codes to enhance the systems' storage efficiency. However, a relatively unexplored aspect of the usage of erasure codes in storage systems -both in data center as well as peer-to-peer environments- is that of the time required for inserting the data (along with the necessary redundancy for fault-tolerance and durability).

When using replication, a source node aiming to store new data can upload one replica of this data to the first storage node, which can concurrently forward the same data to a second storage node, and so on. By applying such a pipelining mechanism, the load for redundancy insertion can be shared, and the source node does not need to upload any redundant information itself. Replication thus naturally supports "in-network" generation of redundancy, that is, generation of new redundancy within the network, through data exchange among storage nodes, which in turn leads to fast insertion of data. In contrast, in erasure encoded systems, the source node 2 is the only one responsible for computing and uploading all the encoded redundant data to the corresponding storage nodes. This has traditionally been the case, because typically the coding process is centralized in nature, and hence all encoded data fragments are generated at a single node, which then also bears the load of inserting the encoded fragments at other storage nodes. The amount of data the source node uploads is then considerably larger, including the data object and its corresponding redundancy, resulting in lower data insertion throughput. Insertion throughput may further be exacerbated when the source node and the set of storage nodes have additional (mismatched) temporal constraints on resources availability, in which case in-network redundancy generation can provide partial mitigation.

We elaborate the effect of temporal resource (un)availability issues with two distinct example scenarios, which we also use later in our experiments to determine how (much) in-network redundancy generation may improve the data insertion throughput:

\footnotetext{
${ }^{2}$ In this paper we call the node which has a full copy of the data, and is primarily responsible for inserting the data redundantly in the rest of the system as the 'source node', irrespectively of the origin and ownership of the data.
} 
(i) In datacenters, storage nodes might be used for computation processes which require efficient access to local disks. Since backup processes consume large amounts of local disk I/O, system administrators might want to avoid backup transfers while nodes are executing I/O intensive tasks - e.g., Mapreduce tasks.

(ii) In peer-to-peer settings, users exchange some of their spare disk resources in order to realize a collaborative data backup service. Such resource sharing may furthermore be driven by other constraints such as trust or friendship. However, desirable users may be online at different times of the day, complicating the data insertion process.

In both cases the insertion of new redundancy by the source node is restricted to the periods when the availability windows of the source overlap that of the storage nodes.

Unlike replication, where in-network redundancy generation is achieved trivially, traditional erasure codes are not easily amenable. Our solution is based on new classes of erasure codes designed for better repair efficiency, obtained through local repairability, that is the property of being able to repair a failure by contacting only a small number of live nodes (e.g. [18, 21]). In particular, to provide a concrete instance that will be used later for experimentation, we will focus on a novel family of erasure codes called SelfRepairing Codes (SRC) [18, whose salient property is that the encoded data stored at each node can be easily regenerated by using information from typically only two live storage nodes. As we will show, local repairability is the key property to achieve in-network redundancy generation. However, SRC have strict constraints on how storage nodes can combine their data to generate content for other nodes, which, along with the temporal availability constraints of nodes, complicate the design of efficient in-network redundancy generation.

In this paper we provide an analytical framework to define valid transfer schedules for the in-network redundancy generation using SRC. We then identify the main requirements that valid scheduling algorithms must satisfy, and show that determining optimal schedules is computationally intractable. Accordingly, we explore several heuristic algorithm implementations which aim at maximizing the utilization of the spare resources of the storage nodes, thus improving the backup throughput. We determine the efficacy of our approach in different environments by using Google data center availability and workload traces, and availability traces from real friend-to-friend (F2F) 24] and peer-to-peer (P2P) applications [26]. Our results show that for data center traces the algorithms proposed for the in-network redundancy generation can increase the throughput of the storage process up to $90 \%$ as compared to classical naive storage approaches, while only requiring $50 \%$ more of the network resources, but using it only when it is otherwise unused. For $\mathrm{P} 2 \mathrm{P} / \mathrm{F} 2 \mathrm{~F}$ traces the throughout can increase up to $60 \%$ while requiring $38 \%$ additional (but again, spare) network resources.

The main contributions of this paper can be summarized as follows:

(i) We introduce the concept of in-network redundancy generation for reducing data insertion latency in erasure code based storage systems, and demonstrate its feasibility using one specific instance of locally repairable code, namely self-repairing codes.

(ii) We define an analytical framework to explore valid data transfer schedules where the in-network redundancy generation process maximizes the use of the available network resources. 
(iii) We show that besides requiring a node availability prediction, determining the optimal data insertion schedule is computationally intractable.

(iv) We propose a set of heuristics for efficient in-network redundancy generation.

(v) We determine the efficacy of in-network redundancy generation in diverse distributed storage environments using real workload and availability trace driven simulations.

The rest of the paper is organized as follows. In Section 2 we provide the background on erasure codes and self-repairing codes, a particular instance of locally repairable codes. In Section 3 we define our in-network redundancy generation process and in Section 4 we show how to schedule the redundancy generation to speedup the insertion of new data. Due to the complexity of determining optimal schedules, we propose in Section 5 several heuristic scheduling algorithms, which are evaluated in Section 6 using real availability traces. Finally in Section 7 we state our conclusions and further research directions.

\section{Background}

The P2P research community has long studied the applicability of erasure codes in low availability environments with limited storage capacity [13, 27]. The growing interest in applying erasure codes in data centers is more recent, and aims at reducing the storage costs [28, 7, 6, 19, 3]. It further triggered a line of research around repairability of storage systems, i.e., how lost redundancy can be replenished, which includes the application of network coding [12, 25] to carry out the repair process in a decentralized manner, designing novel codes with inherent local repairability [18, 21, as well as engineering solutions such as applying multiple levels of encoding on the same [5] or even across different data objects [4].

While fault-tolerance, storage overhead, repairability, but also I/O and bandwidth are well recognized critical bottlenecks for the storage of huge amounts of data, existing literature does not explore yet how data insertion can be optimized in the context of erasure codes based storage. This work leverages on one of these recent approaches on distributed storage systems repairability, namely, novel codes with local repairability, in order to improve the very process of data insertion.

In the following, we provide some background on erasure codes as classically used for distributed storage, as well as on one specific instance of locally repairable codes, Self-Repairing Codes (SRC), which we use in the rest of the paper to demonstrate the feasibility and quantify the benefits of in-network redundancy generation in erasure code based distributed storage systems.

\subsection{Erasure codes for distributed storage}

A classical $\langle n, k\rangle$ erasure code allows to redundantly encode an object of size $M$ into $n$ redundant fragments of size $M / k$, each to be stored in a different storage node. The data storage overhead (or redundancy factor) is then given by $n / k$, and the stored object can be reconstructed by downloading an amount of data equal to $M$, from $k$ or more different nodes out of $n$.

One of the main drawbacks of using classical erasure codes for storage is that redundant fragments can

only be generated by applying coding operations on the original data. The generation of new redundancy is then restricted to nodes that possess the original object (or a copy), namely: the source node, storage nodes that previously reconstructed the original object, or possibly were storing a copy (as is the case in a hybrid model where a full copy of the object is kept, together with encoded fragments). When the original 
raw object is not available, repairing a single node failure consequently entails downloading an amount of information equivalent to the size of the original object, causing a significant communication overhead.

In order to mitigate this communication overhead, a new family of erasure codes called Regenerating Codes (see e.g. [12, 25] and reference therein) was recently designed by adopting ideas from network coding [1], a popular mechanism deployed to improve the throughput utilization of a given network topology. The main advantage of Regenerating Codes is that new redundant fragments can be generated by downloading an amount of data $\beta$ from $d$ other redundant fragments, where $d \geq k$, and $\beta \leq M / k$. Unlike in classical erasure codes, Regenerating Codes can thus repair missing fragments by downloading only an amount of data equal to $d \beta$, where usually $d \beta \ll M$. However, the maximum communication savings occur for large values of $d$, in which cases however, the chance to find $d$ available nodes might be very low, limiting the practicality of such codes.

\subsection{Homomorphic Self-Repairing Codes (HSRC)}

Self-Repairing Codes (SRC) [18] are a new family of erasure codes designed to minimize the maintenance overhead by reducing the number of nodes $d$ required to be contacted to recreate lost fragments. A specific family of SRC, named Homomorphic Self-Repairing codes (HSRC), has the property that two encoded fragments can be xored for such a regeneration, i.e. $d=2$, as long as not more than half of the nodes have failed. This locally repairable property makes HSRC suitable for the in-network redundancy generation since partial redundant data stored in two different nodes can be used to generate data for a third node, without requiring the intervention of the source node. However, as we will show below, the pairs of nodes used for that purpose cannot be arbitrary chosen.

Let us recall briefly the construction of HSRC. We denote finite fields by $\mathbb{F}$. The cardinality of $\mathbb{F}$ is given by its index, that is, $\mathbb{F}_{2}$ is the binary field with two elements (the two bits 0 and 1 ), and $\mathbb{F}_{q}$ is the finite field with $q$ elements. If $q=2^{m}$, for some positive integer $m$, we can fix a $\mathbb{F}_{2}$-basis of $\mathbb{F}_{q}$ and represent an element $\mathbf{x} \in \mathbb{F}_{2^{m}}$ using an $m$-dimensional vector $\mathbf{x}=\left(x_{1}, \ldots, x_{m}\right)$ where $x_{i} \in \mathbb{F}_{2}, i=1, \ldots, m$.

Let $\mathbf{o}$ be the object to be stored over a set of $n$ nodes, which is represented as a data vector of size $k \times m$ bits, $k \leq m$, i.e.:

$$
\mathbf{o}=\left(o_{1}, \ldots, o_{k}\right), \quad o_{i} \in \mathbb{F}_{2^{m}}
$$

Given these $k$ original elements, the $n$ redundant fragments are obtained by evaluating the polynomial

$$
p(X)=\sum_{i=1}^{k} o_{i} X^{2^{(i-1)}} \in \mathbb{F}_{2^{m}}[X]
$$

in $n$ non-zero values $\alpha_{1}, \ldots, \alpha_{n}$ of $\mathbb{F}_{2^{m}}$, yielding the redundant vector $\mathbf{r}$ of size $n \times m$ bits, i.e.:

$$
\mathbf{r}=\left(r_{1}, \ldots, r_{n}\right), \quad r_{i}=p\left(\alpha_{i}\right) \in \mathbb{F}_{2^{m}}
$$

In particular we need the code parameters $\langle n, k\rangle$ to satisfy

$$
1<k<n \leq 2^{m}-1
$$

\section{HSRC Redundancy Generation}

The main important property of HSRC is its homomorphic property. From [18] we have that:

Lemma 1 Let $a, b \in \mathbb{F}_{2^{m}}$ and let $p(X)$ be the polynomial defined in (11), then $p(a+b)=p(a)+p(b)$. 
This implies that we can generate a redundant element $r_{k}=p\left(\alpha_{k}\right)$ from $r_{i}=p\left(\alpha_{i}\right)$ and $r_{j}=p\left(\alpha_{j}\right)$ if and only if $\alpha_{k}=\alpha_{i}+\alpha_{j}$. This homomorphic property is one way of obtaining local repair by contacting only $d=2$ nodes.

Example 1 Consider a $\langle n=7, k=3\rangle$ HSRC and an object $\mathbf{o}=\left(o_{1}, o_{2}, o_{3}\right)$ of size $3 \times 4$ bits, where $o_{i} \in \mathbb{F}_{2^{4}}$, $i=1,2,3$. We write $o_{1}=\left(o_{11}, o_{12}, o_{13}, o_{14}\right), o_{2}=\left(o_{21}, o_{22}, o_{23}, o_{24}\right), o_{3}=\left(o_{31}, o_{32}, o_{33}, o_{34}\right)$, from which we compute $p(X)=\sum_{i=1}^{k} o_{i} X^{2^{i-1}}$. We evaluate $p(X)$ in $n=7$ values of $\mathbb{F}_{2^{4}}$, represented in vector form as $\alpha_{1}=(1,0,0,0), \alpha_{2}=(0,1,0,0), \alpha_{3}=(1,1,0,0), \alpha_{4}=(0,0,1,0), \alpha_{5}=(1,0,1,0), \alpha_{6}=(0,1,1,0)$, $\alpha_{7}=(1,1,1,0)$, yielding:

$$
\begin{aligned}
& r_{1}=p\left(\alpha_{1}\right)=\left(o_{11}+o_{21}+o_{31}, o_{12}+o_{22}+o_{32}, o_{13}+o_{23}+o_{33}\right), \\
& r_{2}=p\left(\alpha_{2}\right)=\left(o_{14}+o_{23}+o_{31}+o_{34}, o_{11}+o_{14}+o_{23}+o_{24}+o_{31}+o_{32}+\right. \\
& \left.o_{34}+o_{12}+o_{21}+o_{24}+o_{232}+o_{33}, o_{13}+o_{22}+o_{33}+o_{34}\right), \\
& r_{3}=p\left(\alpha_{3}\right)=\left(o_{11}+o_{21}+o_{31}+o_{14}+o_{23}+o_{31}+o_{34}, o_{12}+o_{22}+o_{11}+o_{14}+o_{23}+o_{24}+\right. \\
& \left.o_{31}+o_{34}+o_{13}+o_{23}+o_{12}+o_{21}+o_{24}+o_{32}, o_{14}+o_{24}+o_{13}+o_{22}+o_{33}\right), \\
& r_{4}=p\left(\alpha_{4}\right)=\left(o_{13}+o_{24}+o_{21}+o_{31}+o_{33}, o_{13}+o_{14}+o_{21}+o_{22}+o_{24}+o_{32}+o_{33}+o_{34}+\right. \\
& \left.o_{11}+o_{14}+o_{22}+o_{23}+o_{31}+o_{33}+o_{34}, o_{12}+o_{23}+o_{24}+o_{32}+o_{34}\right) \text {, } \\
& r_{5}=p\left(\alpha_{5}\right)=\left(o_{11}+o_{13}+o_{24}+o_{33}, o_{12}+o_{13}+o_{14}+o_{21}+o_{24}+o_{33}+o_{34}+\right. \\
& \left.o_{11}+o_{13}+o_{14}+o_{22}+o_{31}+o_{34}, o_{12}+o_{14}+o_{23}+o_{32}\right), \\
& r_{6}=p\left(\alpha_{6}\right)=\left(o_{14}+o_{23}+o_{34}+o_{13}+o_{24}+o_{21}+o_{33}, o_{11}+o_{23}+o_{31}+o_{13}+o_{21}+o_{22}+o_{33}+o_{12}+o_{21}+\right. \\
& \left.o_{24}+o_{32}+o_{11}+o_{14}+o_{22}+o_{23}+o_{31}+o_{34}, o_{13}+o_{22}+o_{33}+o_{12}+o_{23}+o_{24}+o_{32}\right), \\
& r_{7}=p\left(\alpha_{7}\right)=\left(o_{11}+o_{14}+o_{23}+o_{34}+o_{13}+o_{24}+o_{31}+o_{33}, o_{11}+o_{23}+o_{31}+o_{13}+o_{21}+\right. \\
& o_{12}+o_{33}+o_{32}+o_{12}+o_{21}+o_{24}+o_{32}+o_{11}+o_{14}+o_{22}+o_{33}+o_{31}+ \\
& \left.o_{34}+o_{13}, o_{13}+o_{22}+o_{33}+o_{12}+o_{23}+o_{34}+o_{32}+o_{14}\right)
\end{aligned}
$$

with corresponding redundant vector

$$
\mathbf{r}=\left(r_{1}, \ldots, r_{7}\right)
$$

We can check that

$$
\begin{aligned}
& p\left(\alpha_{7}\right)=p\left(\alpha_{1}\right)+p\left(\alpha_{6}\right)=p\left(\alpha_{2}\right)+p\left(\alpha_{5}\right)=p\left(\alpha_{3}\right)+p\left(\alpha_{4}\right), \\
& p\left(\alpha_{6}\right)=p\left(\alpha_{1}\right)+p\left(\alpha_{7}\right)=p\left(\alpha_{2}\right)+p\left(\alpha_{4}\right)=p\left(\alpha_{3}\right)+p\left(\alpha_{5}\right),
\end{aligned}
$$

which illustrates the local repairability of the code. Note that we have not used the vector $\alpha_{8}=(0,0,0,1)$ here, which would have resulted in a longer code $n>7$.

We now discuss how HSRC operate in two different scenarios: (i) when the source introduces data in the system, and (ii) during the in-network redundancy generation.

\subsection{Source Redundancy Generation}

The homomorphic property described in Lemma 1 1 has been introduced to repair node failures, though it can similarly serve to generate redundancy from the source. Recall from Lemma 1 that $p(a+b)=p(a)+p(b)$, 
where both $a, b$ can be seen as $m$-dimensional binary vectors, by fixing a $\mathbb{F}_{2}$-basis of $\mathbb{F}_{2^{m}}$. Let us denote this basis by $\left\{b_{1}, \ldots, b_{m}\right\}$. Thus $a$ can be written as $a=\sum_{i=1}^{m} a_{i} b_{i}, a_{i} \in \mathbb{F}_{2}$, and by virtue of the homomorphic property, we get that

$$
p(a)=p\left(\sum_{i=1}^{m} a_{i} b_{i}\right)=\sum_{i=1}^{m} a_{i} p\left(b_{i}\right) .
$$

This means that the source only needs to compute $p\left(b_{1}\right), \ldots, p\left(b_{m}\right)$ for a given basis $\left\{b_{1}, \ldots, b_{m}\right\}$, after which all the other encoded fragments are obtained by xoring pairs of elements in $\left\{p\left(b_{1}\right), \ldots, p\left(b_{m}\right)\right\}$. Thus, when using an $\langle n, k\rangle$ HSRC, the source computes $k(k \leq m)$ encoded fragments $r_{1}=p\left(\alpha_{1}\right), \ldots, r_{k}=p\left(\alpha_{k}\right)$, where $\alpha_{1}, \ldots, \alpha_{k}$ are linearly independent, for example, $\left\{\alpha_{1}, \ldots, \alpha_{k}\right\} \subset\left\{b_{1}, \ldots, b_{m}\right\}$, and then performs the corresponding xoring. The source then injects the $n$ encoded fragments in the network.

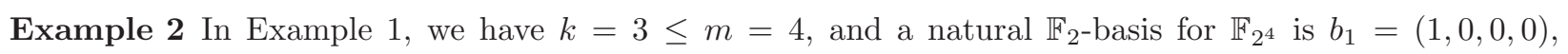
$b_{2}=(0,1,0,0), b_{3}=(0,0,1,0), b_{4}=(0,0,0,1)$. The source can generate redundancy by first computing $r_{1}=p\left(\alpha_{1}\right)=p\left(b_{1}\right), r_{2}=p\left(\alpha_{2}\right)=p\left(b_{2}\right), r_{4}=p\left(\alpha_{4}\right)=p\left(b_{3}\right)$, then $r_{3}=p\left(\alpha_{3}\right)=p\left(\alpha_{1}\right)+p\left(\alpha_{2}\right), r_{5}=p\left(\alpha_{5}\right)=$ $p\left(\alpha_{1}\right)+p\left(\alpha_{4}\right), r_{6}=p\left(\alpha_{6}\right)=p\left(\alpha_{2}\right)+p\left(\alpha_{4}\right)$ and $r_{7}=p\left(\alpha_{7}\right)=p\left(\alpha_{1}\right)+p\left(\alpha_{6}\right)$. The $n=7$ encoded fragments are then ready to be sent over the network. Further notice that the set $B=\left\{p\left(\alpha_{1}\right), p\left(\alpha_{2}\right), p\left(\alpha_{4}\right)\right\}$ can be seen as a basis for the set of redundant fragments, since they are linearly independent, and can be combined to generate every redundant fragment.

\subsection{In-Network Redundancy Generation}

Let us now consider the case where the source might not inject the whole set of $n$ encoded fragments, but only a subset $\left\{r_{i}, i \in I \subset\{1, \ldots, n\}\right\}$ of the encoded fragments. We use the triplet notation $(i, j) \vdash k$ to represent the possibility to generate the element $r_{k}$ by xoring $r_{i}$ and $r_{j}, r_{k}=r_{i}+r_{j}$. Note that due to the commutativity property of the additive operator, triplets $(i, j) \vdash k$ and $(j, i) \vdash k$ can be indistinguishably used to denote the same redundancy generation process. We denote by $\mathcal{C}$ the set with all the feasible repair triplets from a set of $n$ redundant elements. Finally, let us define the following two sets:

Definition 1 (out-creation set) Let $O(i)$ be the set of all the possible $(i, j) \vdash k$ triplets where fragment $r_{i}$ is used to generate some other fragment:

$$
O(i)=\left\{(i, j) \vdash k \mid j=1, \ldots, n, j \neq i, k \text { s.t. } r_{k}=r_{i}+r_{j}\right\} .
$$

Definition 2 (in-creation set) Let $I(k)$ be the set of all the possible $(i, j) \vdash k$ triplets that can be used to create $r_{k}$ :

$$
I(k)=\left\{(i, j) \vdash k \mid r_{k}=r_{i}+r_{j} ; i, j=1, \ldots, n\right\} .
$$

Finally, given a number of redundant elements $n=2^{t}-1$, for any positive integer $t \leq m$, we have from [18, that:

$$
\begin{aligned}
& |O(i)|=n-1 \\
& |I(k)|=(n-1) / 2 .
\end{aligned}
$$


Example 3 In Example 1, we have that

$$
\begin{aligned}
O(1) & =\{(1,3) \vdash 2,(1,2) \vdash 3,(1,5) \vdash 4,(1,4) \vdash 5,(1,7) \vdash 6,(1,6) \vdash 7\}, \\
I(7) & =\{(1,6) \vdash 7,(2,5) \vdash 7,(3,4) \vdash 7\} .
\end{aligned}
$$

\subsection{HSRC: Practical Implementation}

Previously we detailed how to encode a data vector $\mathbf{o}$ of size $k \times m$ bits into a redundant vector $\mathbf{r}$ of size $n \times m$ bits. We showed that HSRC allow this encoding by using data from the source node as well as by using data from other storage nodes. In this subsection we describe one method to practically implement HSRC to encode larger data objects of size $M$, where $M>k \times m$.

The first step to encode an object of size $M$ is to split it into $u=M /(k \times m)$ vector 3 of size $k \times m$ bits. Let us represent the object to be encoded as $\mathbf{o}=\left(o_{1}, \ldots, o_{M}\right)$. After the splitting process, $\mathbf{o}=\left(\overline{\mathbf{o}}_{1}, \ldots, \overline{\mathbf{o}}_{u}\right)$, where $\overline{\mathbf{o}}_{i}=\left(o_{k(i-1)+1}, \ldots, o_{k(i-1)+k}\right), o_{k(i-1)+j} \in \mathbb{F}_{2^{m}}, j=1, \ldots, k$. Each of these vectors $\overline{\mathbf{o}}_{i}$ is individually encoded using the polynomial (11) to obtain an encoded vector $\overline{\mathbf{r}}=\left(\overline{\mathbf{r}}_{1}, \ldots, \overline{\mathbf{r}}_{u}\right)$, with $\overline{\mathbf{r}}_{i}=\left(r_{i, 1}, \ldots, r_{i, n}\right)$, $i=1, \ldots, u$. Finally, the vector $\mathbf{r}=\left(\mathbf{r}_{1}, \ldots, \mathbf{r}_{n}\right)$ with the $n$ fragments to be stored in the system is obtained by concatenating individual elements of $\overline{\mathbf{r}}$ so that $\mathbf{r}_{i}=\left(r_{1, i}, \ldots, r_{u, i}\right)$, namely $\mathbf{r}_{i}$ contains those coefficients $r_{j, i}$ of $\overline{\mathbf{r}}$ that have $i$ as second index.

Example 4 Consider the $\langle n=7, k=3\rangle$ HSRC and the object $\mathbf{o}=\left(o_{1}, \ldots, o_{9}\right)$, where $o_{i} \in \mathbb{F}_{2}$. We split the object into $u=3$ vectors $\mathbf{o}=\left(\overline{\mathbf{o}}_{1}, \overline{\mathbf{o}}_{2}, \overline{\mathbf{o}}_{3}\right)$, where $\overline{\mathbf{o}}_{1}=\left(o_{1}, o_{2}, o_{3}\right), \overline{\mathbf{o}}_{2}=\left(o_{4}, o_{5}, o_{6}\right)$ and $\overline{\mathbf{o}}_{3}=\left(o_{7}, o_{8}, o_{9}\right)$. After encoding each of the individual vectors we obtain the set of redundant vectors $\overline{\mathbf{r}}=\left(\overline{\mathbf{r}}_{1}, \ldots, \overline{\mathbf{r}}_{3}\right)\left(\overline{\mathbf{o}}_{i}\right.$ is encoded to obtain $\overline{\mathbf{r}}_{i}$ ), where $\left|\overline{\mathbf{r}}_{1}\right|=\left|\overline{\mathbf{r}}_{2}\right|=\left|\overline{\mathbf{r}}_{3}\right|=7$. Finally, we can obtain $\mathbf{r}_{2}=\left(\overline{\mathbf{r}}_{1,2}, \overline{\mathbf{r}}_{2,2}, \overline{\mathbf{r}}_{3,2}\right)$, and similarly for all the fragments.

Remark 1 Note that this encoding technique allows stream encoding. As soon as the source node receives the first $k \times m$ bits to store 4 it can generate the vector $\overline{\mathbf{o}}_{1}$, encode it to $\overline{\mathbf{r}}_{1}$, and distribute $\overline{\mathbf{r}}_{1,1}, \ldots, \overline{\mathbf{r}}_{n, 1}$ to the $n$ storage nodes. Similarly, when a storage node receives $\overline{\mathbf{r}}_{i, 1}$ it can forward it to other nodes for in-network redundancy generation, for instance, when the source does not have adequate bandwidth to upload all the $n$ redundant fragments.

Remark 2 To implement computationally efficient codes one can set $m=8$, or $m=32$, for which addition can simply be done by xoring system words, and for which efficient arithmetic libraries are available [20].

In the rest of this paper we will assume that HSRC are implemented using the method described here. We will use the term redundant fragment to refer to each of the redundant elements $\mathbf{r}_{1}, \ldots, \mathbf{r}_{n}$, i.e., each node stores one redundant fragment. And similarly we will use the term redundant chunk to refer to each of the sub-elements $\overline{\mathbf{r}}_{1, i}, \ldots, \overline{\mathbf{r}}_{u, i}$ stored in each node $i$, i.e., each node $i$ can store up to $u$ redundant chunks.

\section{Scheduling the In-Network Redundancy Generation}

In-network redundancy generation has the potential to speedup the insertion of new data in distributed storage systems. However, the magnitude of actual benefit depends on two factors: (i) the availability pattern

\footnotetext{
${ }^{3}$ We assume that the object size $M$ is multiple of $k \times m$, otherwise the object can be encoded by parts and/or zero-padded to meet this requirement.

${ }^{4} \mathrm{~A}$ gateway node in a data center receiving data from a web application end user would be treated as the source node in our model. In such scenarios, the source itself may not be in possession of the whole data in advance.
} 
of the source and storage nodes, which determines the achievable throughput, and (ii) the specific schedule of data transfer among nodes subject to the constraints of resource availability, which determines the actual achieved throughput for data backup. In this section, we explore the scheduling problem, demonstrating that finding an optimal schedule is computationally very expensive even with a few simplifying assumptions, and accordingly motivate some heuristics instead.

Let $s$ be a source node aiming to store a new data object to $n$ different storage nodes, and let $i, i=1, \ldots, n$, represent each of these $n$ storage nodes. We model our system using discrete time steps of duration $\tau$, where at each time step nodes can be available or unavailable to send/receive redundant data. The binary variable $a(i, t) \in\{0,1\}$ denotes this availability for each node $i$ for the corresponding time step $t$. Using this binary variable we can define the maximum amount of data that node $i$ can upload during time step $t$ upload capacity) by

$$
u(i, t)=a(i, t) \cdot \omega_{i} \uparrow(t) \cdot \tau,
$$

where $\omega_{i} \uparrow(t)$ is the upload bandwidth of node $i$ during time step $t$. Similarly, the amount of data each node can download during time step $t$ (download capacity) is given by

$$
d(i, t)=a(i, t) \cdot \omega_{i} \downarrow(t) \cdot \tau,
$$

where $\omega_{i} \downarrow(t)$ represents the download bandwidth of node $i$ during time step $t$.

Then, we define the in-network redundancy generation network as a weighted temporal directed graph $G=(E(t), V(t)), t \geq 0$, with the set of nodes $V(t) \subset\{s, 1,2, \ldots, n\}$, and the set of edges $E(t)=\{(i, j) \mid i, j \in$ $V(t)\}$. The amount of data that nodes might send among themselves is a mapping $f: E(t) \rightarrow \mathbb{R}^{+}$, denoted by $f(i, j, t), \forall(i, j) \in E(t), t \geq 0$.

HSRC characteristics constrain the mapping $f$ since nodes can only send or receive data trough valid redundancy creation triplets:

$$
\begin{aligned}
& \nexists c \in \mathcal{C} \text { s.t. } c=(i, j) \vdash k \Rightarrow f(i, k, t)=0 . \\
& \exists c \in \mathcal{C} \text { s.t. } c=(i, j) \vdash k \Rightarrow f(i, k, t) \geq 0 .
\end{aligned}
$$

Furthermore, we assume (for algorithmic simplicity) that nodes send data through each of the redundancy generation triplets symmetrically:

$$
R(c, t)=f(i, k, t)=f(j, k, t), \forall c \in \mathcal{C} ; c=(i, j) \vdash k .
$$

For ease of notation we will refer to the data sent through each of the redundancy generation triplets simply by $R(c, t)$.

Similarly, because of the upload/download bandwidth constraints, the mapping $f$ must also satisfy the following constraints:

- The amount of data the source uploads is constrained by its upload capacity:

$$
\sum_{i=1}^{n} f(s, i, t) \leq u(s, t) ; \forall i \in V(t)
$$

- The amount of data storage nodes upload is also constrained by their upload capacity:

$$
\sum_{c \in O(i)} R(c, t) \leq u(i, t) ; \forall i \in V(t) .
$$


- The amount of data storage nodes download is restricted by their download capacity:

$$
f(s, i, t)+2 \sum_{c \in I(i)} R(c, t) \leq d(i, t) ; \forall i \in V(t) .
$$

A Bandwidth-Valid In-Network Redundancy Generation Scheduling is any mapping $f$ on $G$ that satisfies the constraints defined in equations (4), (5), (6), (7) and (8).

\subsection{Optimal Schedule}

Let $\theta(i, t)$ be the amount of data that node $i$ had received at the end of time step $t$. For sufficiently large enough files and small values of $m$ (e.g. $m=8$ ), we can assume without loss of generality that $\theta(i, t) / m$ corresponds to the index of the last redundant chunk received by node $i$. Let $\widehat{M}(\bar{t})$ denote the size of the largest possible file that a schedule $f$ can store in $\bar{t}$ time steps, which represents a data insertion throughput of $\widehat{M}(\bar{t}) /(\bar{t} \tau)$. Then, by definition of erasure codes, to consider that a file of size $\widehat{M}(\bar{t})$ has been successfully stored after $\bar{t}$ time steps, each node must receive an amount of data equal to $\widehat{M}(\bar{t}) / k$. Using this fact we can define $\widehat{M}(\bar{t})$ as:

$$
\widehat{M}(\bar{t})=\min (\theta(1, \bar{t}), \ldots, \theta(n, \bar{t})) \times m k .
$$

For a given network $G$ and a duration $\bar{t}$, an in-network redundancy generation scheduling $f$ is then optimal if it maximizes $\widehat{M}(\bar{t})$. Note that to maximize $\widehat{M}(\bar{t})$ an optimal schedule will tend to even out the amount of data $\theta(i, \bar{t})$ sent to each node, obtaining a maximum insertion throughput and a minimum consumption of network resources when $\theta(1, \bar{t})=\cdots=\theta(n, \bar{t})$. We can measure the overall network traffic required by any schedule $f$, after $\bar{t}$ time steps, namely $T(f, \bar{t})$, by:

$$
T(f, \bar{t})=\sum_{t=0}^{\bar{t}}\left(\sum_{i=1}^{n} f(s, i, t)+2 \sum_{c \in \mathcal{C}} R(c, t)\right) .
$$

Accordingly, we define an in-network redundancy generation schedule $f$ to be an optimal minimum-traffic schedule if besides maximizing $\widehat{M}(\bar{t})$, it also minimizes $T(f, \bar{t})$.

From (10) we also want to note that in the total traffic required by the schedule $f, T(f, \bar{t})$, the amount of redundant data each node $i$ receives comes from two distinct components (the two summands within parentheses): $f(s, i, t)$ and $R(c, t)$, where $c=\left(j^{\prime}, j^{\prime \prime}\right) \vdash i$. The first component represents the redundant data inserted by the source while the second component represents the redundant data created through the innetwork redundancy generation process. Since in the last case there are two nodes (i.e., $j^{\prime}$ and $j^{\prime \prime}$ ) uploading data to node $i$ the value has two be multiplied by two, leading to the following remark:

Remark 3 The new redundant data created through the in-network redundancy generation process requires twice the traffic required by the source redundancy generation.

However, although the use of the in-network redundancy generation increases the required network traffic, it has the potential to increase the opportunities of redundancy generation, and hence, increase the data insertion throughput. In Section [6 we will show that the relative increase of insertion throughput surpass the relative increase of network traffic, demonstrating the practicality of the in-network redundancy generation process and its scalability. 

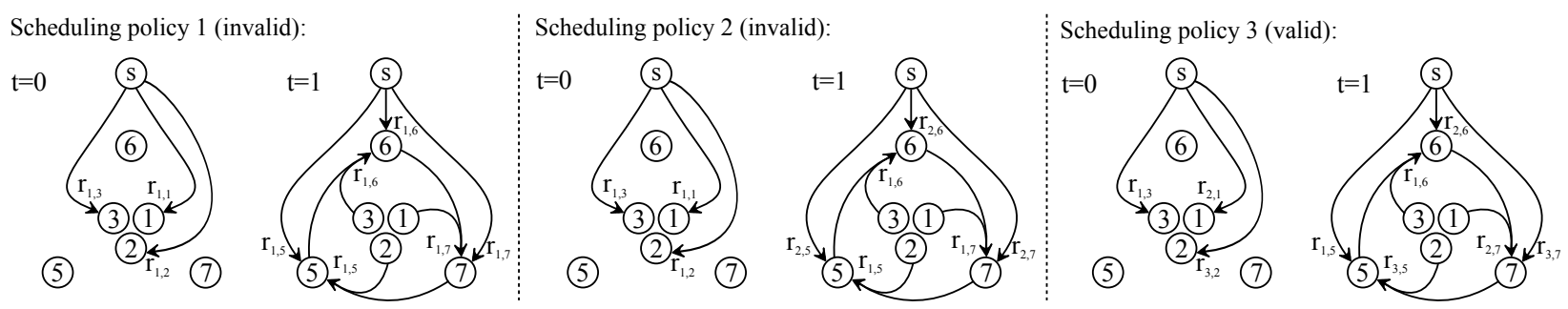

Figure 1: Example of 3 different in-network redundancy scheduling policies for a system where the source node can only upload data concurrently to 3 different nodes. Although the 3 different schedules satisfy the constraints, only scheduling policy 3 is valid.

\subsection{Additional Scheduling Constraints}

In this section we elaborate that while being a bandwidth-valid schedule is a necessary condition, it is not a sufficient condition for the schedule to be actually valid. For that, we will use Example 4 an in-network redundancy generation network using HSRC with parameters $\langle n=7, k=3\rangle$, where the redundant fragments $\mathbf{r}_{1}, \ldots, \mathbf{r}_{7}$ have to be stored in nodes $1, \ldots, 7$ respectively. Recall also that each redundant fragment $\mathbf{r}_{i}$ is composed of 3 redundant chunks, hence $\left|\mathbf{r}_{i}\right|=3$. For ease of notation we will assume that each redundancy generation triplet $c=(i, j) \vdash k, c \in \mathcal{C}$, satisfies the property $k=i \oplus j$ where $\oplus$ denotes the bitwise xor operation. Based on Example 4 we consider three different scheduling policies, all depicted in Figure 1 We assume that due to the limited upload capacity of the source node it can only upload three redundant fragments simultaneously.

In the first scenario, at time $t=0$ the source node sends to nodes 1,2 and 3 their first redundant chunk; at time time $t=1$ it does the same for nodes 5,6 and 7 . Note that if at time step $t=1$ the mapping $f$ tries to make use of the in-network redundancy generation triplets $(1,6) \vdash 7,(2,7) \vdash 5$ and $(3,5) \vdash 6$; nodes 5,6 and 7 end up receiving the same redundant fragment twice. In this case the in-network redundancy traffic does not contribute in speeding up the backup process and only consumes communication resources. Although avoiding this problem is implicit in the definition of a minimum-traffic scheduling, it needs to be explicitly considered during the scheduling.

Consider a second scheduling policy trying to solve the previous problem by sending to nodes 5,6 and 7 the second chunk instead of the first. It allows these nodes to receive two different fragments by time $t=1$. However, it appears a circular dependency problem with triplets $(1,6) \vdash 7,(2,7) \vdash 5$ and $(3,5) \vdash 6$. To show this dependency, imagine that we want to generate fragment $\mathbf{r}_{6,1}$ using non-source data. Note that $\mathbf{r}_{6,1}$ requires of $\mathbf{r}_{5,1}$, and $\mathbf{r}_{5,1}$ requires of $\mathbf{r}_{7,1}$, which at the same time requires of the fragment we aim to generate, $\mathbf{r}_{6,1}$. Although it is a bandwidth-valid schedule, the circular dependency problem makes it an unfeasible schedule.

Finally, in the third case we see how the circular dependency problems can be avoided if the source sends uncorrelated fragments at each time step. It is easy to see from this example that a valid schedule needs to be not only bandwidth-valid, but also ensure that: (i) nodes do not receive duplicated data, and (ii) circular triplet dependencies are prevented.

\subsection{Complexity Analysis}

We show that finding an optimal schedule satisfying all the previous requirements is computationally very expensive, even under further simplifying assumptions: 


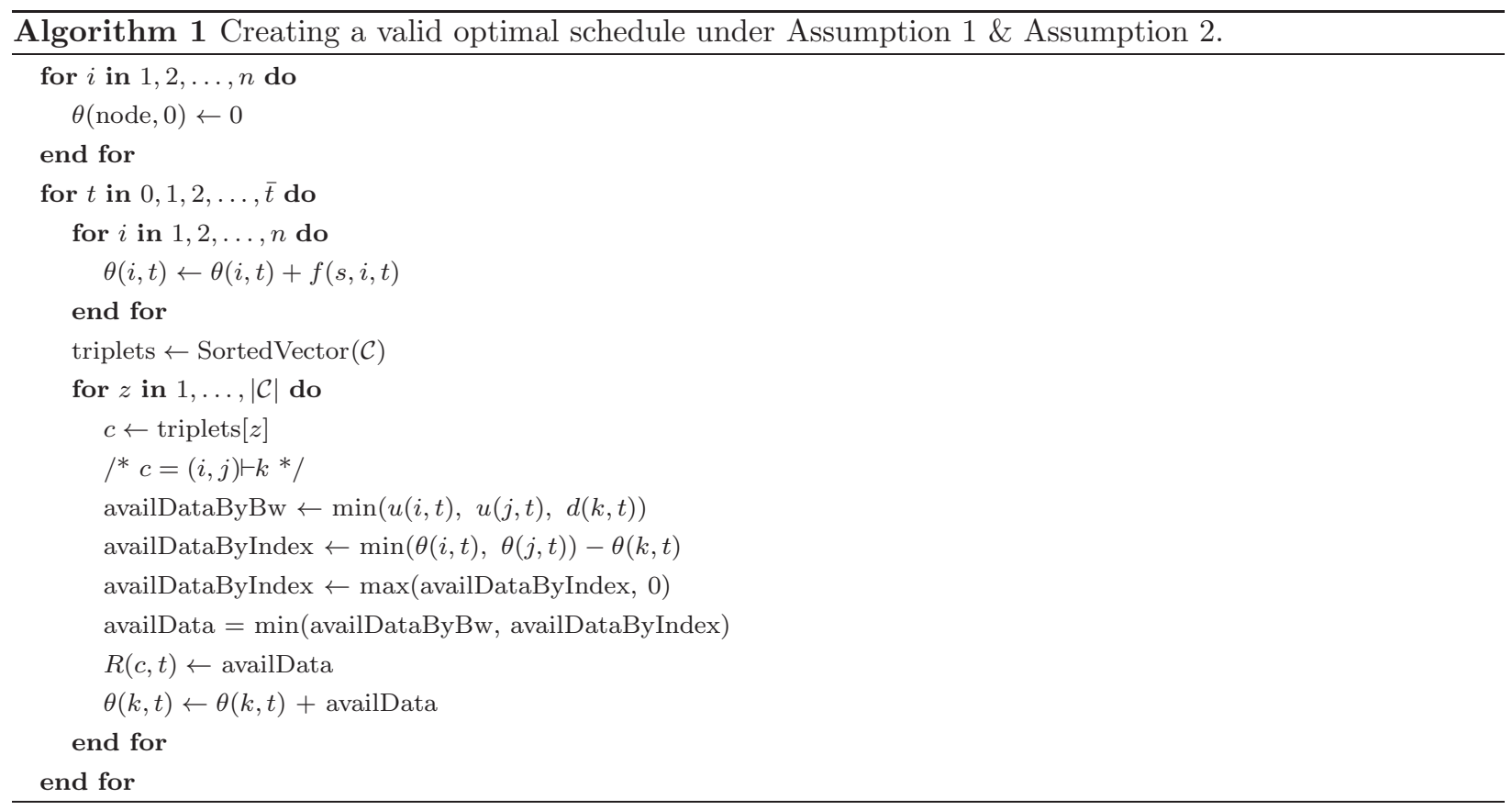

Assumption 1 The amount of data that the source node $s$ sends during each time step t to any storage node $i, f(s, i, t)$, is a constant value and is not part of the optimization problem.

Assumption 2 Storage nodes can only receive redundant chunks sequentially. It means that node $i$ will never receive chunk $\mathbf{r}_{j+1, i}$ before previously receiving chunk $\mathbf{r}_{j, i}$.

It is easy to see that the simplified problem subject to these two assumptions corresponds to a specific instance of the generic case described above. The interesting property about this simplified version of the problem is that we can reduce the decision of choosing the optimal schedule $f$ to an algorithm "SortedVector" which sorts $\mathcal{C}$, as it is shown in Algorithm 1 . It is also easy to see, how due to the iterative use of redundancy generation triplets, Algorithm 1 avoids both the "duplicate data" and the "circular dependencies" problems. However, since $|\mathcal{C}|=n(n-1)$, it means that there are $(n(n-1))$ ! possible ways of sorting $\mathcal{C}$, and thus, $\bar{t} \times(n(n-1))$ ! different scheduling possibilities. Thus, a brute force algorithm to determine the best schedule would have a $O(n !)$ cost.

If we focus on a single time step $t$, then the scheduling problem can be restated as how to choose the best permutation of $\mathcal{C}$. We can represent this decision problem using a permutation tree as is depicted in Figure 2 The weight of the edges in this permutation tree correspond to the negative amount that choosing each edge contributes to $\widehat{M}(t)$. Choosing the best scheduling algorithm tis the same than finding the shortest path between vertices $v$ and $d$ in the permutation tree. The Bellman-Ford algorithm can find the shortest past with cost $O(|E| \cdot|V|)$ where $|E|$ and $|V|$ respectively represent the number of edges and vertices in the permutation tree. However, in our permutation tree the number of edges and vertices are both $(n(n-1))$ !, which makes finding the optimal schedule for even the simplified problem computationally exorbitantly expensive, even for small number of nodes $n$. Hence, we consider the general problem described in 4.1 to be also intractable. 


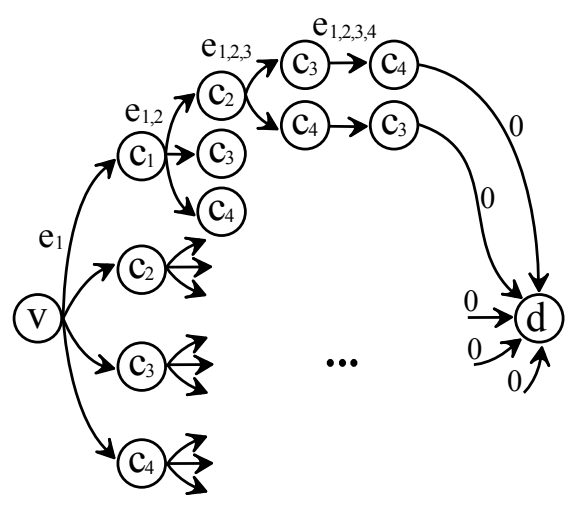

Figure 2: Example of a permutation tree to implement "SortedVector" in Algorithm 1] We assume in this example a hypothetical system where $|\mathcal{C}|=4$.

\section{$5 \quad$ Heuristic Scheduling Algorithms}

In this section we investigate several heuristics for scheduling the in-network redundancy generation. We split the scheduling problem into two parts, following the strategy presented in Algorithm 1,

The heuristics do not require Assumption 1, thus allowing the source node to send different amounts of data to each storage node. We however still rely on Assumption 2, which allows us to model the decision problem with a sorting algorithm, as previously outlined in Algorithm 1. Thus, the overall scheduling problem is decomposed into the following two decisions: (i) How does the source node schedule its uploads? (ii) How are redundancy generation triplets sorted?

\subsection{Scheduling traffic from the source}

Recall that generating redundancy directly from the source node involves less bandwidth than doing it with in-network techniques (Remark 3). Thus, a good source traffic scheduling should aim at maximizing the source's upload capacity utilization. Furthermore, the schedule must also try to ensure that the source injected data can be further used for the in-network redundancy generation.

Given a $\langle n, k\rangle$ HSRC, where $n=2^{k}-1$, any subset of $k$ linearly independent encoded fragments forms a basis, denoted by $B$ (see Example 2 for an illustration). Let $\mathcal{B}$ be the set of all the possible bases $B$. Since each storage node stores one redundant fragment, we use $\widehat{\mathcal{B}}(t)$ to represent all the basis of $\mathcal{B}$ whose corresponding storage nodes are available at a time step $t$ (and likewise, refer to each combination of such nodes as an available basis):

$$
\widehat{\mathcal{B}}(t)=\{B \in \mathcal{B} \mid a(i, t)=1, \quad \forall i \in B\} .
$$

From the set of available basis, $\widehat{\mathcal{B}}(t)$, the source node selects one basis $B$ and uploads some data to each node $i \in B$. The amount of data the source uploads to each node $i \in B$ is set to guarantee that at the end of time step $t$, all these nodes have received the same amount of data, $\theta(i, t)=\theta(j, t), \forall i, j \in B$. From equations (9) and (10) we know that evening out the data all nodes receives allows to minimize network traffic and maximize insertion throughput. To even out the amount of data each node in basis receives and maximizing the utilization of the upload capacity 5 of the source, the source needs to send to each node $i \in B$

\footnotetext{
${ }^{5}$ We assume that the upload capacity of the source is less than the download capacity of the basis nodes.
} 
an amount of data equal to

$$
\frac{1}{|B|}\left(u(s, t)+\sum_{j \in B} \theta(j, t-1)\right)-\theta(i, t) .
$$

Besides determining how to distribute the upload capacity of the source between nodes of a basis $B$, the source node also needs to select the basis $B$ from all the available ones $\widehat{\mathcal{B}}(t$ ) (if more than one basis is available). We consider the following heuristic policies for the source node to select a specific basis $B$ :

- Random: $B$ is randomly selected from $\mathcal{B}(t)$. Repeating this procedure for several time steps is expected to ensure that all nodes receive approximately the same amount of data from the source.

- Minimum Data: The source selects the basis $B$ that on an average has received less redundant data. It means that $B$ is the basis that minimizes $\frac{1}{k} \sum_{i \in B} \theta(i, t)$. This policy tries to homogenize the amount of data all nodes receive.

- Maximum Data: The source selects the basis $B$ that on an average has received more redundant data. It means that $B$ is the basis that maximizes $\frac{1}{k} \sum_{i \in B} \theta(i, t)$. This policy tries to have a basis of nodes with enough data to allow the in-network redundancy generation for the entire data object even when the source may not be available.

- No Basis: The source does not considers any basis and instead uploads data to all the online nodes. The upload bandwidth of the source is also distributed to guarantee that, after time step $t$, all online nodes have received the same amount of data.

\subsection{Sorting the redundancy generation triplets}

At each time step $t$, once the source allocates its upload capacity to nodes from a specific available basis $B$, the remaining upload/download capacity of the available nodes is used for in-network redundancy generation. For that purpose, the list of available triplets, $\widehat{\mathcal{C}}(t)$, is determined as follows:

$$
\widehat{\mathcal{C}}(t)=\{(i, j) \vdash k \in \mathcal{C} \mid a(i, t)=a(j, t)=a(k, t)=1, \quad \forall(i, j) \vdash k \in B\}
$$

Then, the set of available triplets $\widehat{\mathcal{C}}(t)$ is sorted, and the available upload/download capacity of storage nodes allocated according to this priority (i.e., the first available triplets have more preference). We consider the following sorting heuristics:

- Random: Repair triplets are randomly sorted. This policy tries to uniformly distribute the utilization of network resources to maximize the amount of in-network generated data.

- Minimum Data: The list of available triplets are sorted in ascending order according to the amount of data $\theta(k, t)$ the destination 6 node $k$ has received. This policy tries to prioritize the redundancy generation in those nodes that have received less redundant data.

- Maximum Data: Similarly to the Minimum Data policy, however, triplets are sorted in descending order. This policy tries to maximize the amount of data some specific subset of nodes receive, to allow them to sustain the redundancy generation process even when the source is not available.

\footnotetext{
${ }^{6}$ Node $k$ is the destination of a triplet $c, c=(i, j) \vdash k$.
} 


\begin{tabular}{ccc}
\hline Policy Name & Source Policy & In-Network Policy \\
\hline RndFlw & random & maximum flow \\
\hline RndDta & random & minimum data \\
\hline MinFlw & minimum data & maximum flow \\
\hline MinDta & minimum data & minimum data \\
\hline
\end{tabular}

Table 1: Different policy combinations.

- Maximum Flow: The triplets are sorted in descending order according to the amount of redundant data these nodes can help generate. Note that the amount of data a triplet $c$ can generate at each time step $t$, where $c=(i, j) \vdash k$, is given by:

$$
\begin{gathered}
\min (u(i, t), u(j, t), d(k, t), \\
\theta(i, t)-\theta(k, t), \\
\theta(j, t)-\theta(k, t))
\end{gathered}
$$

This policy tries to maximize the amount of new redundancy generated per time step.

\section{$6 \quad$ Experimental results}

He have proposed four different policies for the source traffic scheduling problem and four policies for the triplets sorting problem. However, after an extensive experimental evaluation of all polices we will only report for each case the two best policies (in terms of achieved throughput). At the source, the random and minimum data policies consistently outperform the others, and at the storage nodes, the maximum flow and minimum data sorting policies for the triplets likewise outperform the others. We will refer to each of the combinations as denoted in Table 1 . First, the interpretation of the good performance of the random policy in the source node is that the use of random bases favors the diversity of information among the nodes, which in turn enables more redundancy generation triplets. Second, it is interesting to note that the minimum data policy obtains good storage throughput in both cases, which leads us to infer that in general, prioritizing redundancy generation in those nodes that have received less data is a good strategy to maximize the throughput of the backup process.

\subsection{Setting}

We considered a $\langle n=7, k=3\rangle$-HSRC, which is a code that can achieve a static data resiliency similar to a 3 -way replication, but requiring only a redundancy factor of $7 / 3 \simeq 2.33$. 18 Using this erasure code we simulated various backup processes with different node (un)availability patterns for a fixed number of time steps $\bar{t}$. In all the simulated cases we consider three different metrics:

(i) The maximum amount of data that can be stored in $\bar{t}$ time steps, $\widehat{M}(\bar{t})$.

(ii) The amount of data the source node uploads per unit of useful data backed up,

$$
\frac{1}{\widehat{M}(\bar{t})} \sum_{t=0}^{\bar{t}} \sum_{i=1}^{n} f(s, i, t) .
$$


(iii) The total traffic generated per unit of useful data stored, $T(f, \bar{t}) / \widehat{M}(\bar{t})$.

We evaluate the three metrics for a system using an in-network redundancy generation algorithm and we compare our results with a system using the naive erasure coding backup process, where the source uploads all the data directly to each storage node. Our results depict the savings and gains, in percentage, of using an in-network redundancy algorithm with respect to the naive approach.

Regarding the (un)availability patterns of nodes and their bandwidth constraints we consider two different distributed storage cases:

(i) A P2P-like environment where we assume, to simplify simulations, that nodes have an upload bandwidth uniformly distributed between $20 \mathrm{Kbps}$ and $200 \mathrm{Kbps}$, and an asymmetric download bandwidth equal to four times their upload bandwidth. Nodes in this category follow two different availability traces from real decentralized application: (i) traces from users of an instant messaging (IM) service 24] and traces from P2P nodes in the aMule KAD DHT overlay [26. In both cases we filter the nodes that on average stay online more than 4, 6 and 12 daily hours, obtaining different mean availability scenarios.

(ii) Real availability traces collected from a Google datacentel 7 . The traces contain the normalized I/O load of more than 12,000 servers monitored for a period of one month. We consider that a server is available to upload/download data when its I/O load is under the $p$-percentile load. We consider three different percentiles, $p=0.25,0.5,0.75$, giving us three different node availability constraints.

Finally the time step duration is set to $\tau=1$ hour and we obtain the results by averaging the results of 500 backup processes of $\bar{t}=120$ time steps each (5 days).

Before discussing the results, we will like to note that the experiments make a few simplifying assumptions. Furthermore, real deployments have somewhat different workload characteristics than what have been considered above. Hence, the quantitative results we report are only indicative (and many more settings could possibly be experimented), and instead the specific choices help us showcase the potential benefits of our approach in only a qualitative manner.

\subsection{Results}

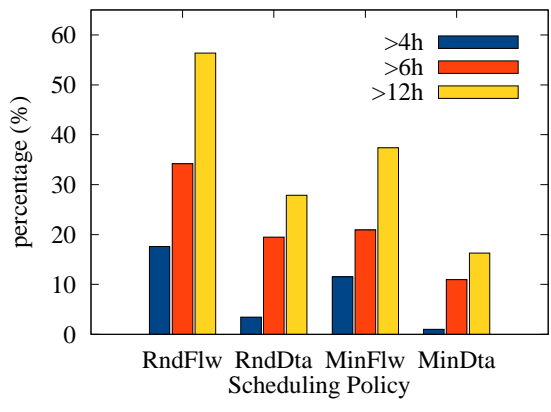

(a) IM traces.

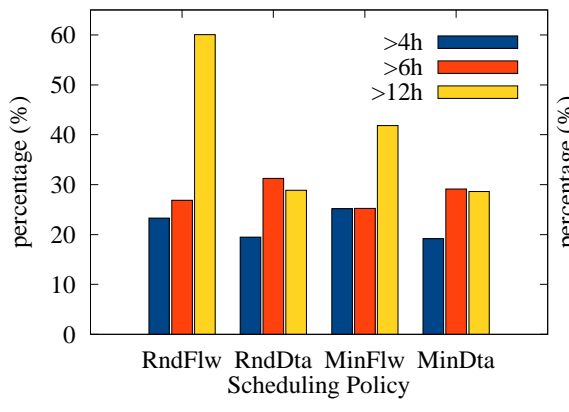

(b) P2P traces.

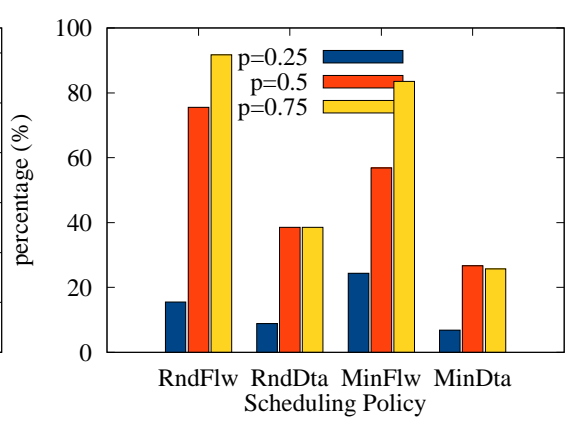

(c) Google traces.

Figure 3: Increment of the maximum amount of stored data (throughput) for the three different availability traces.

\footnotetext{
${ }^{7}$ Publicly available at: http://code.google.com/p/googleclusterdata/
} 
Storage Throughput. In Figure 3 we show the increment of the data insertion throughput achieved by the in-network redundancy generation process. We can see how the gain is higher when nodes are more available for redundancy generation. This fact is a consequence of the constraint in eq. (5) requiring redundancy generation triplets to be symmetric, which requires the three involved nodes in each triplet to be available simultaneously. The higher the online availability, the higher the chances to find online three nodes from a triplet. Further, we observe that the RndFlw policy achieves significantly better results in comparison to other policies; the second best policy is MinFlw. It is easy then to see that the Maximum Flow heuristic plays an important role on the overall redundancy generation throughput, which tries to maximize the use of those nodes that can potentially generate more redundancy. Additionally, a Random source selection policy provides more benefits than the Minimum Data policy.

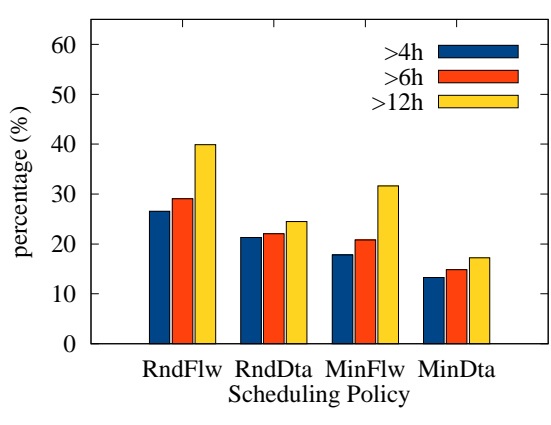

(a) IM traces.

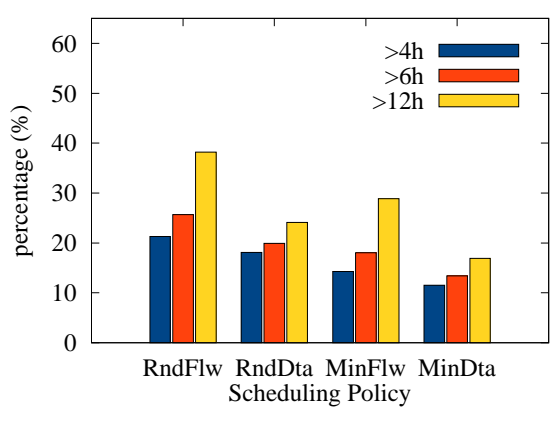

(b) P2P traces.

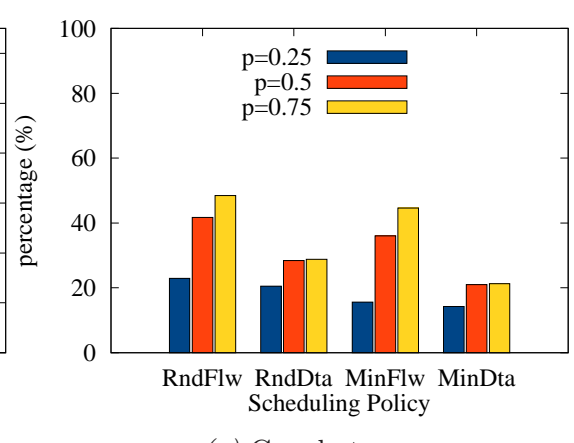

(c) Google traces.

Figure 4: Increment of the required network traffic of the in-network redundancy generation strategy for the three different availability traces.

Network Traffic. In Figure 4 we show the increment on the required network traffic of the in-network redundancy generation strategy as compared to the traditional redundancy generation. As noted previously (in Remark 31), the total traffic required for in-network redundancy generation can be up to twice the needed by the traditional process (i.e. $100 \%$ traffic increment). However, since the in-network redundancy generation cannot always take place due to the node availability constraints, the traffic increment is always below $100 \%$. As it is expected then, the traffic increment is minimized when nodes are less available, in which case the source has to generate and introduce larger amounts of redundancy (i.e., less reduction in the data uploaded by source, as shown in Figure (5). It is also important to note that the increase in traffic is approximately the same or even less than the increase in storage throughput even for low availability scenarios. Thus the in-network redundancy generation scales well by achieving a better utilization of the available network resources than the classical storage process.

Data Uploaded by the Source. In Figure 5 we show the reduction of data uploaded by the source. In the traditional approach, the source needs to upload $7 / 3 \simeq 2.33$ times the size of the actual data to be stored; $4 / 7 \simeq 57 \%$ of this data is redundant, however the in-network redundancy generation process allows to reduce the amount of data uploaded by the source. In this figure we can see how in the best case (RndFlw policy) our approach reduces the source's load by $40 \%$ (out of a possible $57 \%$ ), yielding $40-60 \%$ increase in storage throughput.

Finally, we want to note that the in-network redundancy performance requires finding three available 


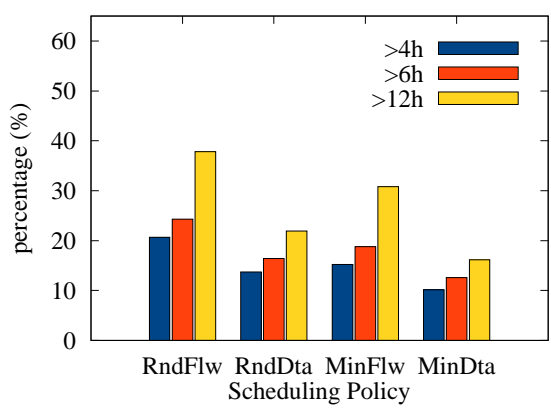

(a) IM traces.

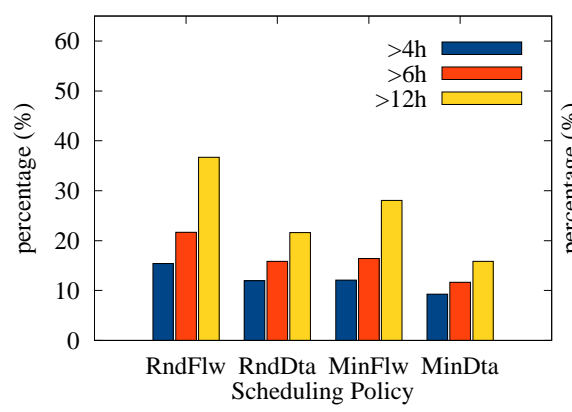

(b) P2P traces.

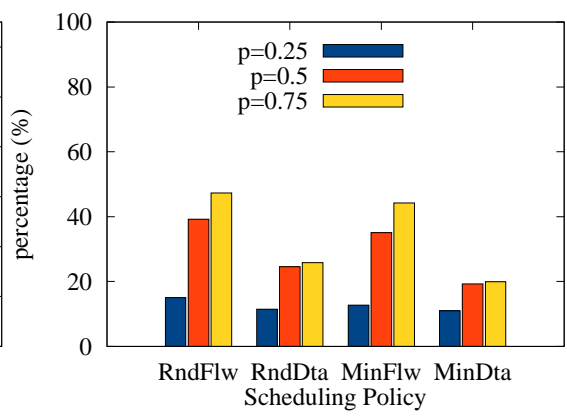

(c) Google traces.

Figure 5: Reduction of the data uploaded by the source for the three different availability traces.

nodes simultaneously, which becomes difficult on environments with fewer backup opportunities. To solve this problem, we would need to look at more sophisticated in-network redundancy generation strategies not subjected to the symmetric constraint (defined in eq. (5)), so that nodes can forward and store partiallygenerated data. However, the scheduling problem will be much more complicated, and is beyond the reach of this first work. Furthermore, in real traces, nodes will have correlation (e.g., based on batch jobs), which are missing in the synthetic traces, and such correlations can be leveraged in practice. Exploring both these aspects will be part of our future work.

\section{Conclusions}

In this work we propose and explore how storage nodes can collaborate among themselves to generate erasure encoded redundancy by leveraging novel erasure codes' local-repairability property. Doing so not only reduces a source node's load to insert erasure encoded data, but also significantly improves the overall throughput of the data insertion process. We demonstrate the idea using self-repairing codes. We show that determining an optimal schedule among nodes to carry out in-network redundancy generation subject to resource constraints of the system (nodes and network) is computationally prohibitive even under simplifying assumptions. However, experiments supported by real availability traces from a Google data center, and $\mathrm{P} 2 \mathrm{P} / \mathrm{F} 2 \mathrm{~F}$ applications show that some heuristics we propose yield significant gain in storage throughput under these diverse settings, proving the practicality of not only the idea in general, but also that of the specific proposed heuristics.

\section{References}

[1] Ahlswede, R., Cai, N., Li, S.R., and Yeung, R.W. "Network Information Flow." IEEE Transactions on Information Theory, 46(4), 2000.

[2] Borthakur, D. "The Apache Hadoop Distributed File System." http://hadoop.apache.org/hdfs/, 2011.

[3] Calder, B., Wang, J., Ogus, A., Nilakantan, N., Skjolsvold, A., McKelvie, S., Xu, Y., Srivastav, S., Wu, J., Simitci, H., Haridas, J., Uddaraju, C., Khatri, H., Edwards, A., Bedekar, V., Mainali, S., Abbasi, R., Agarwal, A., Haq, M.F.u., Haq, M.I.u., Bhardwaj, D., Dayanand, S., Adusumilli, A., McNett, M., 
Sankaran, S., Manivannan, K., and Rigas, L. "Windows Azure Storage: a highly available cloud storage service with strong consistency." In ACM Symposium on Operating Systems Principles (SOSP). 2011.

[4] Datta, A. and Oggier, F. "Redundantly Grouped Cross-object Coding for Repairable Storage." In Asia-Pacific Workshop on Systems (APSys). 2012.

[5] Duminuco, A. and Biersack, E.W. "Hierarchical Codes: How to Make Erasure Codes Attractive for Peer-to-Peer Storage Systems." In Intl. Conference on Peer-to-Peer Computing (P2P). 2008.

[6] Fan, B., Tantisiriroj, W., Xiao, L., and Gibson, G. "DiskReduce: RAID for data-intensive scalable computing." In Annual Workshop on Petascale Data Storage (PDSW). 2009.

[7] Ford, D., Labelle, F., Popovici, F.I., Stokely, M., Truong, V.A., Barroso, L., Grimes, C., and Quinlan, S. "Availability in globally distributed storage systems." In USENIX conference on Operating systems design and implementation (OSDI). 2010.

[8] Ghemawat, S., Gobioff, H., and Leung, S. "The Google File System." In ACM Symposium on Operating Systems Principles (SOSP). 2003.

[9] Gu, Y. and Grossman, R.L. "Sector: A high performance wide area community data storage and sharing system." Future Generation Comp. Syst., 26(5):720-728, 2010.

[10] Hastorun, D., Jampani, M., Kakulapati, G., Pilchin, A., Sivasubramanian, S., Vosshall, P., and Vogels, W. "Dynamo: Amazon's Highly Available Key-value Store." In Symposium on Operating Systems Principles (SOSP). 2007.

[11] Huang, C., Simitci, H., Xu, Y., Ogus, A., Calder, B., Gopalan, P., Li, J., and Yekhanin, S. "Erasure coding in windows azure storage." In USENIX Annual Technical Conference (ATC). 2012.

[12] Kermarrec, A.M., Scouarnec, N.L., and Straub, G. "Repairing Multiple Failures with Coordinated and Adaptive Regenerating Codes." In Intl. Symposium on Network Coding (NETCOD). 2011.

[13] Kubiatowicz, J., Bindel, D., Chen, Y., Czerwinski, S., Eaton, P., Geels, D., Gummadi, R., Rhea, S., Weatherspoon, H., Weimer, W., Wells, C., and Zhao, B. "OceanStore: An Architecture for GlobalScale Persistent Storage." In Intl. Conference on Architectural Support for Programming Languages and Operating Systems (ASPLOS). 2000.

[14] Lakshman, A. and Malik, P. "The Apache Cassandra Project." http://cassandra.apache.org/, 2011.

[15] Li, J. and Dabek, F. "F2F: reliable storage in open networks." In Intl. Workshop on Peer-To-Peer Systems (IPTPS). 2006.

[16] Lin, W.K., Chiu, D.M., and Lee, Y.B. "Erasure code replication revisited." In Intl. Conference on Peer-to-Peer Computing (P2P). 2004.

[17] Liu, S., Schulze, J.P., Herr, L., Weekley, J.D., Zhu, B., van Osdol, N., Plepys, D., and Wan, M. "CineGrid Exchange: A workflow-based peta-scale distributed storage platform on a high-speed network." Future Generation Comp. Syst., 27(7):966-976, 2011. 
[18] Oggier, F. and Datta, A. "Self-repairing Homomorphic Codes for Distributed Storage Systems." In Intl. Conference on Computer Communications (INFOCOM). 2011.

[19] Pawar, S., Noorshams, N., Rouayheb, S.E., and Ramchandran, K. "DRESS Codes for the Storage Cloud: Simple Randomized Constructions." In IEEE Intl. Symposium on Information Theory (ISIT). 2011.

[20] Plank, J.S., Simmerman, S., and Schuman, C.D. "Jerasure: A Library in C/C ++ Facilitating Erasure Coding for Storage Applications." Technical Report CS-08-627, University of Tennessee, 2008.

[21] Rawat, A. and Vishwanath, S. "On Locality in Distributed Storage Systems." arXiv preprint arXiv:1204.6098, 2012.

[22] Reed, I. and Solomon, G. "Polynomial Codes Over Certain Finite Fields." Journal of the Society for Industrial and Applied Mathematics, 8(2):300-304, 1960.

[23] Rodrigues, R. and Liskov, B. "High Availability in DHTs: Erasure Coding vs. Replication." In Intl. Workshop on Peer-To-Peer Systems (IPTPS). 2005.

[24] Sharma, R., Datta, A., Dell Amico, M., and Michiardi, P. "An empirical study of availability in friendto-friend storage systems." In Intl. Conference on Peer-to-Peer Computing (P2P). 2011.

[25] Shum, K. "Cooperative Regenerating Codes for Distributed Storage Systems." In IEEE Intl. Conference on Communications (ICC). 2011.

[26] Steiner, M., En-Najjary, T., and Biersack, E. "A Global View of KAD." In ACM SIGCOMM conference on Internet Measurement (IMC). 2007.

[27] Weatherspoon, H. and Kubiatowicz, J.D. "Erasure Coding vs. Replication: A quantitative Comparison." In Intl. Workshop on Peer-To-Peer Systems (IPTPS). 2002.

[28] Zhang, Z., Deshpande, A., Ma, X., Thereska, E., and Narayanan, D. "Does erasure coding have a role to play in my data center?" Technical Report MSR-TR-2010-52, Microsoft Research, 2010. 